\title{
Genotypes and Phenotypes of Chinese Pediatric Patients with Idiopathic and Heritable Pulmonary Arterial Hypertension: Experiences from a Single Center
}

\author{
Hong-Sheng Zhang, Qian Liu, Chun-Mei Piao, Yan Zhu, \\ Qiang-Qiang Li, Jie Du, and Hong Gu
}

\begin{abstract}
The aim of this study was to determine the clinical outcomes of gene mutations in Chinese pediatric patients with idiopathic and heritable pulmonary arterial hypertension. We screened gene mutations in 62 pediatric patients who visited Beijing Anzhen Hospital from 2008 September to 2017 August with targeted exome kits containing 22 pulmonary arterial hypertension-related genes. The clinical and hemodynamic characteristics and outcomes of these patients were retrospectively analyzed. In a cohort of 62 patients, a total of 27 gene mutations were identified with 20 mutations
\end{abstract}

H.-S. Zhang

Department of Pediatrics, Capital Medical University, Beijing, China

Pediatric Cardiology Department, Beijing Anzhen Hospital, Capital Medical University, Beijing, China

Q. Liu

Department of Cardiology, Children's Hospital of Hebei Province,

Shijiazhuang, Hebei, China

C.-M. Piao · J. Du

Beijing Anzhen Hospital, The Key Laboratory of Remodeling-Related Cardiovascular Diseases, Capital Medical University, Beijing, China

Beijing Institute of Heart Lung and Blood Vessel Diseases, Beijing, China e-mail: jiedu@ccmu.edu.cn

Y. Zhu $\cdot$ Q.-Q. Li $\cdot$ H. Gu $(\bowtie)$

Pediatric Cardiology Department, Beijing Anzhen Hospital, Capital Medical University, Beijing, China 
in BMPR2, two mutations in ACVRL1, two mutations in KCNK3 and three mutations in NOTCH3. The average age at diagnosis was $77.5 \pm 53.8$ months. 28 patients (14 mutation carriers) underwent cardiac catherization examinations, with the acute vasodilator testing. Mutation carriers had higher right atrial pressure and tended to have higher pulmonary arterial pressure and pulmonary vascular resistance index than mutation non-carriers. Eight patients responded to acute vasodilator testing and all were mutation non-carriers $(p=0.002)$. The median survival for mutation carriers was 24.0 months. Although similar treatments were employed, mutation carriers had higher mortality rates than mutation non-carriers $(p=0.036)$. The 1-, 2-, 3- year survival rate of mutation non-carriers were $93.6 \%, 90.0 \%$, and $66.9 \%$, respectively, while for mutation carriers, the proportion were $79.8 \%, 49.9 \%$, and $33.3 \%$. In conclusion, early gene screening for pediatric patients with idiopathic pulmonary arterial hypertension and heritable pulmonary arterial hypertension is recommended, and more aggressive treatment for mutation carriers is advisable.

\section{Keywords}

Mutation $\cdot$ hemodynamics $\cdot$ mortality

\subsection{Introduction}

Pulmonary arterial hypertension (PAH) is a progressive pulmonary vessel disease, which can ultimately lead to progressive right-sided heart failure and premature death [1]. Idiopathic pulmonary arterial hypertension (IPAH) is a sporadic form of $\mathrm{PAH}$, which occurs without family aggregation or known etiology [2], while heritable pulmonary arterial hypertension (HPAH) occurs in a familial aggregate. Without adequate treatment, these patients' condition deteriorates rapidly with a median survival time of 2.8 years in adults and 10 months in children [3, 4]. Despite recent advances in management and improvement of prognosis, the pathophysiology of IPAH is not yet explained in detail.

Genetic studies showed that gene mutations in some members of the transforming growth factor- $\beta$ (TGF- $\beta$ ) pathway were associated with heritable PAH, including bone morphogenetic protein receptor type II (BMPR2), activin receptor-like kinase 1 (ALK1), endoglin (ENG) and SMAD family [5-8]. Mutations in ALK1 and ENG are thought to be linked to hereditary hemorrhagic telangiectasia (HHT), an autosomal dominant genetic disorder typically characterized by small arteriovenous malformations [9, 10]. With the development of gene detection technology, more additional gene mutations were found out to be the disease-causing genes, including KNCK3 (potassium two pore domain channel subfamily K member 3), CAV1 (Caveolin 1), NOTCH3, BMPR1B (bone morphogenetic protein receptor, type 1B), EDN1 (endothelin 1), TBX4 (T-box 4), TRPC6 (transient receptor potential cation channel, subfamily $\mathrm{C}$, member 6), TOPBP1 (topoisomerase (DNA) II binding protein 1), and SERPINE1 (serpin family E member 1) [7, 8, 11]. IPAH and HPAH were seen more commonly in pediatric patients $[12,13]$, indicating more genetic factors participate in the pathogenesis of pediatric $\mathrm{PAH}$. 
In addition to the effect on the pathogenicity of PAH, gene mutations have previously been linked to worse clinical outcomes in adult patients [14, 15]. An international multi-center research revealed that patients with childhood IPAH or HPAH with BMPR2 or ACVRL1 mutation have poorer clinical outcomes than mutation non-carriers [16]. However, the study sample was relatively small, and data addressing the relationship of gene mutations with clinical outcomes of pediatric patients is scarce till now.

In this study, we applied a targeted capture exome sequence to investigate the genetic etiology of Chinese pediatric patients with IPAH and HPAH. We aimed to assess the prevalence of gene mutations in this group of patients and to assess the relationship between genotypes and phenotypes.

\subsection{Methods}

\subsection{Selection of Patients}

Sixty-two pediatric patients with IPAH and HPAH who visited Beijing Anzhen Hospital from 2008 September to 2017 August were included in the study. PAH was defined as a mean pulmonary arterial pressure (MPAP) $\geq 25 \mathrm{mmHg}$ with a pulmonary artery wedge pressure $\leq 15 \mathrm{mmHg}$ and pulmonary vascular resistance $>3$ Wood units by cardiac catheterization [1]. Acute vasodilator testing was performed during cardiac catheterization and a positive response was defined according to current guidelines as a decrease in MPAP of at least 10 to $<40 \mathrm{mmHg}$ with a stable cardiac output [17]. Clinical, functional, and hemodynamic characteristics of patients were collected at the time of diagnosis and during the follow-up period. The study procedures were approved by the Research Ethics Committee of Beijing Anzhen Hospital. All patients gave informed consent in accordance with local ethical guidelines.

\subsection{Genetic Studies}

Genomic DNA was isolated using QIAamp Blood Midi kit (Qiagen). Libraries were prepared according to the Illumina standard protocol. Exon and exon-intron junction sequences of 22 candidate genes (ACVRL1, AGTR2, ANGPT1, BMPR2, BMPR1A, BMPR1B, ENG, NOS3, HTR2B, KCNA5, KCNQ1, SLC6A4, SMAD1, SMAD2, SMAD3, SMAD4, SMAD5, SMAD6, SMAD7, TGFB1, TRPC6, THBS1) were enriched using a GenCap custom enrichment kit (My-Genostics). The enriched libraries were sequenced on an Illumina Hi Seq 2000 sequencer for paired reads of $100 \mathrm{bp}$ in length.

\subsection{Statistical Analysis}

Qualitative variables were expressed as frequency and percentage and mean \pm standard deviation and median [interquartile range] for quantitative variables. We 
compared clinical and hemodynamic characteristics between mutation carriers and non-carriers by one-way analysis of variance or the median test for continuous measures and the chi-square test for categorical measures. The Kaplan-Meier survival curve and the Log-rank test were used to assess the difference in survival rates between two groups. A $p$-value less than 0.05 was considered to indicate statistical significance. All analyses were performed with the Statistical Package for Social Sciences for Windows (SPSS, Chicago, IL) version 19.0.

\subsection{Results}

\subsubsection{Clinical Characteristics}

Sixty-two unrelated childhood IPAH or HPAH probands, 26 (41.9\%) female, were included in this study. The average age at diagnosis was $77.5 \pm 53.8$ months. The median interval from onset of symptoms to diagnosis was 4 months (1 48 months). Twenty-eight $(45.2 \%)$ patients were presented with markedly decreased cardiac function (NYHA class III-IV). The average plasma BNP concentration was $1198.8 \pm 2239.9 \mathrm{pg} / \mathrm{mL}$. Of 62 children evaluated, 20 (32.3\%) carried BMPR2 mutations, and $2(3.2 \%)$ carried ALK1 mutations; 2 (3.2\%) carried KCNK3 mutations and $3(4.8 \%)$ carried NOTCH3 mutation. Patients were divided into two groups based on the presence or absence of gene mutations, and clinical and hemodynamic characteristics were compared (details in Table 21.1). Twenty-eight patients, half of them mutation carriers, underwent cardiac catherization examinations, with acute vasodilator testing (details in Table 21.2). Mutation non-carriers had lower right atrial pressure than mutation carriers $(p=0.016)$. Mutation carriers tended to have higher pulmonary arterial pressure and higher pulmonary vascular resistance index than mutation non-carriers although the differences were not statistically significant. Eight patients responded to acute vasodilator challenge testing; all were mutation non-carriers $(p=0.002)$.

Table 21.1 Clinical characteristics of patients at diagnosis

\begin{tabular}{l|l|l|l} 
Variables & Mutation carriers $(n=27)$ & Mutation non-carriers $(n=35)$ & $p$-value \\
\hline Female $(\%)$ & $8(29.6)$ & $18(51.4)$ & 0.120 \\
\hline $\begin{array}{l}\text { Age at diagnosis, months } \\
\text { NYHA function class }\end{array}$ & $80.7 \pm 49.8$ & $77.5 \pm 53.6$ & 0.992 \\
\hline I-II & 15 & 19 & 0.921 \\
\hline III-IV & 12 & 16 & \\
BNP, $\mathrm{pg} / \mathrm{mL}$ & $953.0 \pm 1874.7$ & $1358 \pm 2487.8$ & 0.502 \\
TBIL, $\mu \mathrm{mol} / \mathrm{L}$ & $13.3 \pm 5.7$ & $16.0 \pm 9.4$ & 0.220 \\
\hline DBIL, $\mu \mathrm{mol} / \mathrm{L}$ & $3.3 \pm 1.5$ & $4.0 \pm 2.5$ & 0.214 \\
\hline $\mathrm{UA}, \mu \mathrm{mol} / \mathrm{L}$ & $421.2 \pm 173.0$ & $424.7 \pm 159.3$ & 0.940
\end{tabular}

NYHA New York Heart Association, BNP brain natriuretic peptide, TBIL total bilirubin, DBIL direct bilirubin, $U A$ uric acid 
Table 21.2 Hemodynamic characteristics of patients at diagnosis

\begin{tabular}{l|l|l|l} 
Variables & Mutation carriers, $n=14$ & Mutation non-carriers, $n=14$ & $p$-value \\
\hline PASP, $\mathrm{mmHg}$ & $100.1 \pm 22.9$ & $83.8 \pm 33.4$ & 0.144 \\
$\mathrm{PADP}, \mathrm{mmHg}$ & $59.8 \pm 14.0$ & $45.7 \pm 22.0$ & 0.054 \\
$\mathrm{MPAP}, \mathrm{mmHg}$ & $74.5 \pm 16.2$ & $59.1 \pm 23.3$ & 0.052 \\
$\mathrm{RAP}, \mathrm{mmHg}$ & $10.5 \pm 4.1$ & $7.2 \pm 2.0$ & 0.016 \\
$\mathrm{PVRI}, \mathrm{WU} \cdot \mathrm{m}^{2}$ & $20.3 \pm 11.1$ & $14.1 \pm 9.5$ & 0.130 \\
$\mathrm{CI}, 1 / \mathrm{min} / \mathrm{m}^{2}$ & $3.3 \pm 1.3$ & $3.9 \pm 1.1$ & 0.669 \\
$\mathrm{SvO}, \%$ & $59.3 \pm 11.3$ & $67.2 \pm 6.6$ & 0.564 \\
$\mathrm{AVT}(\%)$ & $0(0)$ & $8(57.1)$ & 0.002
\end{tabular}

$P A S P$ pulmonary artery systolic pressure, $P A D P$ pulmonary artery diastolic pressure, $M P A P$ mean pulmonary arterial pressure, $R A P$ right atrial pressure, $P V R I$ pulmonary vascular resistance index, $W U$ Wood units, $\mathrm{CI}$ cardiac index, $\mathrm{SvO}_{2}$ mixed venous oxygen saturation, $A V T$ acute pulmonary vasodilatation test

Table 21.3 Targeted drugs' protocols of patients

\begin{tabular}{|l|l|l|l|}
\hline Protocols & All, $n=56$ & $\begin{array}{l}\text { Carriers, } \\
n=24\end{array}$ & Non-carriers, $n=32$ \\
\hline Monotherapy (\%) & $29(51.8)$ & $10(41.7)$ & $19(59.4)$ \\
\hline Bosentan & 21 & 8 & 13 \\
\hline Sildenafil & 2 & 0 & 2 \\
\hline Tadalafil & 4 & 0 & 4 \\
\hline Beraprost sodium & 2 & 0 & 2 \\
\hline Dual therapy (\%) & $23(41.1)$ & $11(45.8)$ & $12(37.5)$ \\
\hline Bosentan and sildenafil & 5 & 3 & 2 \\
\hline Bosentan and tadalafil & 10 & 2 & 8 \\
\hline Bosentan and beraprost sodium & 3 & 3 & 0 \\
\hline Ambrisentan and sildenafil & 1 & 1 & 0 \\
\hline Ambrisentan and tadalafil & 1 & 1 & 0 \\
\hline Macitentan and sildenafil & 1 & 1 & 0 \\
\hline Sildenafil and beraprost sodium & 1 & 0 & 1 \\
\hline Vardenafil and beraprost sodium & 1 & 0 & 1 \\
\hline Triple therapy (\%) & $4(7.1)$ & $3(12.5)$ & $1(3.1)$ \\
Bosentan, sildenafil, and iloprost & 2 & 2 & 0 \\
\hline Bosentan, tadalafil, and beraprost sodium & 1 & 0 & 1 \\
\hline Bosentan, beraprost sodium, and treprostinil & 1 & 1 & 0
\end{tabular}

\subsubsection{Targeted Drug Therapy}

Fifty-six patients received PAH-targeted treatment. The utilization of targeted drugs is listed in Table 21.3. Endothelin-receptor antagonists were the medications most commonly used. The percentage of patients who received monotherapy, dual therapy, and triple therapy were $41.7 \%, 45.8 \%$, and $12.5 \%$ in mutation carriers and $59.4 \%, 37.5 \%$, and $3.1 \%$ in mutation non-carriers, respectively. No differences were found between mutation carriers and mutation non-carriers. 


\subsubsection{Outcome of Patients}

The median duration of follow-up was 20.5 months (0-108 months). One patient underwent lung transplantation at the age of 16, after 4 years of targeted drug therapy. Eleven out of 20 BMPR 2 mutation carriers, both of the 2 ALK1 mutation carriers, 1 of 2 KCNK3 mutation carriers, and 9 of 39 mutation non-carriers died during the follow-up period. The median survival for mutation carriers was 24.0 months. The 1-, 2-, 3-year survival of mutation non-carriers were $93.6 \%, 80.0 \%$, and $66.9 \%$, respectively, while for mutation non-carriers, the proportions were 79.8\%, 49.9\%, and 33.3\% (see Fig. 21.1), respectively. The clinical characteristics between the death group and survival group

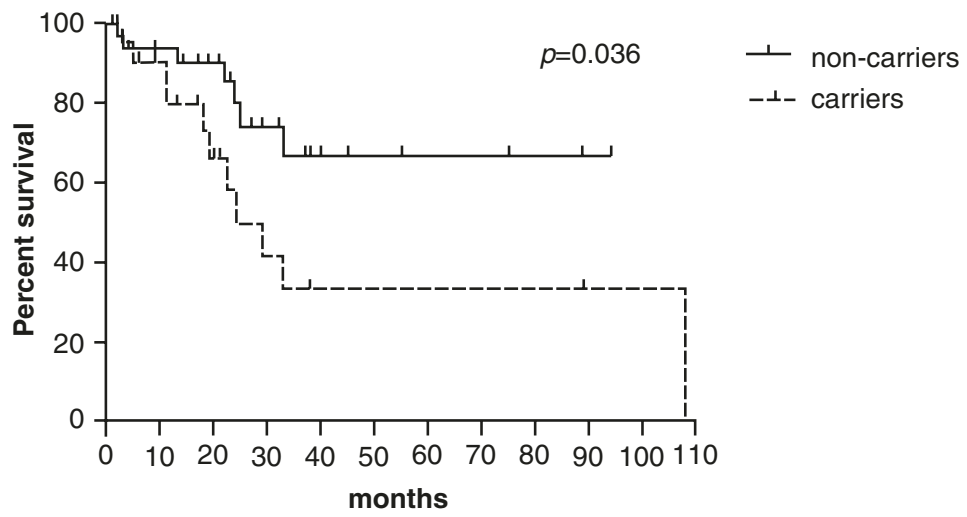

Number at risk:

Carriers

Non-carriers

$\begin{array}{cccccccccccc}23 & 18 & 10 & 6 & 3 & 3 & 3 & 3 & 3 & 2 & 2 & 1 \\ 34 & 27 & 23 & 12 & 7 & 5 & 4 & 4 & 3 & 2 & 1 & 0\end{array}$

Fig. 21.1 Survival rate of mutation carriers and non-carriers with IPAH/HPAH

Table 21.4 Characteristics of dead and surviving patients

\begin{tabular}{|c|c|c|c|}
\hline Variables & Deaths, $n=23$ & Survivors, $n=34$ & $p$-value \\
\hline Female (\%) & $7(30.4)$ & $18(52.9)$ & \\
\hline Age, months & $69.9 \pm 57.6$ & $87.9 \pm 50.0$ & 0.227 \\
\hline NYHA function class & & & 0.849 \\
\hline I-II & 15 & 23 & \\
\hline III-IV & 8 & 11 & \\
\hline Gene mutation, (\%) & $14(60.9)$ & $11(32.4)$ & 0.033 \\
\hline BNP, pg/ml & $1763.9 \pm 3179.5$ & $920.1 \pm 1367.5$ & 0.195 \\
\hline PASP, $\mathrm{mmHg}$ & $97.2 \pm 13.4$ & $89.1 \pm 37.2$ & 0.496 \\
\hline PADP, mmHg & $58.2 \pm 10.0$ & $50.0 \pm 23.6$ & 0.292 \\
\hline MPAP, $\mathrm{mmHg}$ & $75.4 \pm 10.3$ & $63.3 \pm 26.3$ & 0.277 \\
\hline RAP, mmHg & $11.9 \pm 3.8$ & $6.9 \pm 2.1$ & 0.000 \\
\hline PVRI, WU· ${ }^{2}$ & $22.8 \pm 11.5$ & $14.0 \pm 9.5$ & 0.044 \\
\hline $\mathrm{CI}, \mathrm{L} / \mathrm{min} / \mathrm{m}^{2}$ & $2.9 \pm 1.2$ & $4.0 \pm 1.1$ & 0.032 \\
\hline $\mathrm{SvO}_{2}, \%$ & $64.5 \pm 12.5$ & $70.8 \pm 6.2$ & 0.098 \\
\hline
\end{tabular}

NYHA New York Heart Association, BNP brain natriuretic peptide, PASP pulmonary artery systolic pressure, $P A D P$ pulmonary artery diastolic pressure, MPAP mean pulmonary arterial pressure, $R A P$ right atrial pressure, $P V R I$ pulmonary vascular resistance index, $\mathrm{CI}$ cardiac index, $\mathrm{Sv} \mathrm{O}_{2}$ mixed venous oxygen saturation 
were compared (for details, see Table 21.4). The results showed that dead patients had higher RAP, PVRI, and lower cardiac index than the survival group. The mutation of $\mathrm{PAH}-$ related genes is the main risk factor of death $(\mathrm{OR}=6.418,95 \% \mathrm{CI} 1.393,27.123)$.

\subsection{Discussion}

In this study, we retrospectively analyzed the clinical outcomes of gene mutations in Chinese pediatric patients with idiopathic and heritable pulmonary arterial hypertension. Gene mutations are important causes of PAH. The BMPR2 gene encodes a transmembrane serine/threonine kinase receptor of the bone morphogenetic protein (BMP) pathway, which is involved in the regulation of growth and apoptosis of pulmonary smooth muscle cells and pulmonary endothelial cells [18]. Hundreds of BMPR2 mutations have been identified in $70 \%$ of familial PAH patients and up to $40 \%$ of IPAH cases $[12,19]$. In our study, BMPR2 was also the most often mutation-accounting for $32.3 \%$ of all the patients.

Mutations in ALK1 are identified as the major cause of HHT [15]. Neither of the two patients with ALK1 mutations in our study met the diagnostic criteria for HHT. As clinical manifestations of HHT develop with increasing age, the majority of ALK1 mutation carriers might not have clinical evidence of HHT during childhood [15]. Both of the two patients carrying ALK1 mutations died during the follow-up period, suggesting it a rapidly progressing type. A larger sample size is needed for the assessment of ALK1 on phenotype of these patients.

The KCNK3 gene encodes for an outward $\mathrm{K}+$ channel and is a member of twopore-domain $\mathrm{K}+$ channels (K2P) [20]. KCNK3 inhibition participates extensively in the whole spectrum of $\mathrm{PAH}$ pathomechanism from vasoconstriction and vascular cell proliferation to PAH-associated chronic inflammation [21]. NOTCH3 is a transmembrane receptor protein that participates in the modulation of cell proliferation, differentiation, apoptosis, and migration [22]. Compared with that of BMPR2 and ALK1, the research on KCNK3 and NOTCH3 is relatively less. The relationship between these gene mutations and clinical phenotypes needs further verification.

Previous studies on adult patients have suggested that mutation carriers present PAH approximately 10 years earlier than noncarriers, with more compromised hemodynamic status and worse prognosis [14]. Similar results were also obtained from studies on pediatric patients with IPAH and HPAH [16, 23]. In our study, two groups of patients were similar in gender and age, while mutation carriers tended to have higher pulmonary arterial pressure and pulmonary arterial resistance index than mutation non-carriers although the difference was not statistically significant. Moreover, mutation carriers had higher right atrial pressure compared with mutation non-carriers, indicating right heart burden of mutation carriers higher than mutation non-carriers. Similar with the results of previous research, our study showed that pediatric patients with PAH-related gene mutations responded poorly to acute vasodilator testing. No differences were found between two groups in WHO function class and blood biochemical index. As the clinical symptoms of IPAH were atypical, the majority of patients came to our center until obvious symptoms of right heart failure occurred and, thus, blood biochemical index and heart function indexes at baseline could not be used to assess the whole progression of the disease. 
Targeted drugs have benefited the patients with pulmonary hypertension [24, 25]. Targeted drugs were approved in China from 2000s: bosentan in 2005, followed by iloprost, inhaled, in 2007, ambrisentan in 2012, treprostinil in 2014, and tadalafil in 2015. Endothelin receptor antagonists were the most commonly used targeted drugs in our patients, followed by phosphodiesterase inhibitors. More than $90 \%$ of our patients accepted monotherapy or dual therapy in spite of the relatively serious condition. Treatment options were usually made according to the status of the patients, as well as their financial situation. As the only available intravenous prostanoid, the application of treprostinil was limited due to the price and side effects of subcutaneous catheterization.

Our results indicated that the prognosis of pediatric patients with IPAH and HPAH was poor in China. The 3-year survival rate of patients with gene mutations was only $33.3 \%$, with a median survival time of 24 months, even in the modern targeted drugs era. More effort is needed to explore the pathophysiological mechanisms of IPAH and HPAH as well as more effective treatments.

In conclusion, pediatric patients with IPAH and HPAH had worse outcomes when gene mutations exist. Early gene screening for pediatric patients with IPAH and HPAH is recommended, and more aggressive treatment for mutation carriers is advisable.

Acknowledgments We are grateful to the patients and their families whose generosity and cooperation have made this study possible.

Conflicting Interests: The authors declare that there is no conflict of interest.

Funding: This work was supported by the National Natural Science Foundation of China (81570442).

Author Contributions: Hong Gu and Jie Du conceived and designed the research; Hong-sheng Zhang performed all the research. Hong-sheng Zhang wrote the main manuscript text. Qian Liu and Qiang-qiang Li analyzed data; Yan Zhu prepared tables and the figure; Hong-sheng Zhang and Chun-mei Piao edited and revised the manuscript. All authors reviewed the manuscript.

\section{References}

1. Galiè N, Humbert M, Vachiery JL, et al. ESC/ERS guidelines for the diagnosis and treatment of pulmonary hypertension. Kardiol Pol. 2016;73(12):1127.

2. Loyd JE, Atkinson JB, Pietra GG, Virmani R, Newman JH. Heterogeneity of pathologic lesions in familial primary pulmonary hypertension. Am Rev Respir Dis. 1988;138(4):952-7.

3. Runo JR, Loyd JE, hypertension P p. Lancet. 2003;361(9368):1533-44.

4. Barst RJ, Maislin G, Fishman AP. Vasodilator therapy for primary pulmonary hypertension in children. Circulation. 1999;99(9):1197-208.

5. International PPHC, Lane KB, Machado RD, et al. Heterozygous germline mutations in BMPR2, encoding a TGF-beta receptor, cause familial primary pulmonary hypertension. Nat Genet. 2000;26(1):81-4.

6. Machado RD, Pauciulo MW, Thomson JR, et al. BMPR2 haploinsufficiency as the inherited molecular mechanism for primary pulmonary hypertension. Am J Hum Genet. 2001;68(1):92-102.

7. Elliott CG. Genetics of pulmonary arterial hypertension. Clin Chest Med. 2013;34(4):651-63.

8. Garcia-Rivas G, Jerjes-Sanchez C, Rodriguez D, Garcia-Pelaez J, Trevino VA. Systematic review of genetic mutations in pulmonary arterial hypertension. BMC Med Genet. 2017;18(1):82.

9. McAllister KA, Grogg KM, Johnson DW, et al. Endoglin, a TGF-beta binding protein of endothelial cells, is the gene for hereditary haemorrhagic telangiectasia type 1 . Nat Genet. 1994;8(4):345-51. 
10. Johnson DW, Berg JN, Baldwin MA, et al. Mutations in the activin receptor-like kinase 1 gene in hereditary haemorrhagic telangiectasia type 2. Nat Genet. 1996;13(2):189-95.

11. Girerd B, Lau E, Montani D, Humbert M. Genetics of pulmonary hypertension in the clinic. Curr Opin Pulm Med. 2017;23(5):386-91.

12. Hansmann G, Hoeper MM. Registries for paediatric pulmonary hypertension. Eur Respir J. 2013;42(3):580-3.

13. Berger RM, Beghetti M, Humpl T, et al. Clinical features of paediatric pulmonary hypertension: a registry study. Lancet. 2012;379(9815):537-46.

14. Sztrymf B, Coulet F, Girerd B, et al. Clinical outcomes of pulmonary arterial hypertension in carriers of BMPR2 mutation. Am J Respir Crit Care Med. 2008;177(12):1377-83.

15. Girerd B, Montani D, Coulet F, et al. Clinical outcomes of pulmonary arterial hypertension in patients carrying an ACVRL1 (ALK1) mutation. Am J Respir Crit Care Med. 2010;181(8):851-61.

16. Chida A, Shintani M, Yagi H, et al. Outcomes of childhood pulmonary arterial hypertension in BMPR2 and ALK1 mutation carriers. Am J Cardiol. 2012;110(4):586-93.

17. Sitbon O, Humbert M, Jais X, et al. Long-term response to calcium channel blockers in idiopathic pulmonary arterial hypertension. Circulation. 2005;111(23):3105-11.

18. van der Bruggen CE, Happe CM, Dorfmuller P, et al. Bone morphogenetic protein receptor Type 2 mutation in pulmonary arterial hypertension: a view on the right ventricle. Circulation. 2016;133(18):1747-60.

19. Soubrier F, Chung WK, Machado R, et al. Genetics and genomics of pulmonary arterial hypertension. J Am Coll Cardiol. 2013;62(25 Suppl):D13-21.

20. Ma L, Roman-Campos D, Austin ED, et al. A novel channelopathy in pulmonary arterial hypertension. N Engl J Med. 2013;369(4):351-61.

21. Antigny F, Hautefort A, Meloche J, et al. Potassium Channel Subfamily K Member 3 (KCNK3) contributes to the development of pulmonary arterial hypertension clinical perspective. Circulation. 2016;133(14):1371-85.

22. Li X, Zhang X, Leathers R, et al. Notch3 signaling promotes the development of pulmonary arterial hypertension. Nat Med. 2009;15(11):1289-97.

23. Rosenzweig EB, Morse JH, Knowles JA, et al. Clinical implications of determining BMPR2 mutation status in a large cohort of children and adults with pulmonary arterial hypertension. J Heart Lung Transplant. 2008;27(6):668-74.

24. Zhang R, Dai LZ, Xie WP, et al. Survival of Chinese patients with pulmonary arterial hypertension in the modern treatment era. Chest. 2011;140(2):301-9.

25. Rival G, Lacasse Y, Martin S, Bonnet S, Provencher S. Effect of pulmonary arterial hypertension-specific therapies on health-related quality of life: a systematic review. Chest. 2014;146(3):686-708.

Open Access This chapter is licensed under the terms of the Creative Commons Attribution 4.0 International License (http://creativecommons.org/licenses/by/4.0/), which permits use, sharing, adaptation, distribution and reproduction in any medium or format, as long as you give appropriate credit to the original author(s) and the source, provide a link to the Creative Commons license and indicate if changes were made.

The images or other third party material in this chapter are included in the chapter's Creative Commons license, unless indicated otherwise in a credit line to the material. If material is not included in the chapter's Creative Commons license and your intended use is not permitted by statutory regulation or exceeds the permitted use, you will need to obtain permission directly from the copyright holder.

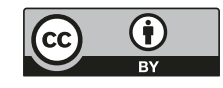

